\title{
Abnormalities of copper accumulation in cell lines established from nine different alleles of mottled are the same as those found in Menkes disease
}

\author{
W Masson, H Hughes, D Papworth, Y Boyd, N Horn
}

\begin{abstract}
Menkes disease (MD) is caused by a defect in copper homeostasis and has a recognised mouse model, mottled $\left(A t p 7 a^{M o}\right)$. Copper uptake and retention assays performed on fibroblast cultures have been used successfully for pre- and postnatal diagnosis of Menkes disease. We report here the results of these assays applied to primary fibroblast cultures established from nine independent mottled alleles associated with phenotypes of varying severity maintained on identical genetic backgrounds. No significant differences were found between the different alleles, or between the mottled cultures and fibroblasts established from MD patients. Thus, in the mouse, the data obtained for copper retention/uptake at the cellular level do not correlate with the severity of the phenotype.
\end{abstract}

$(\Im$ Med Genet 1997;34:729-732)

Keywords: copper; Menkes disease; ATP7A; mottled

Menkes disease (MD) is an X linked copper homeostasis defect in which affected males rarely survive beyond 3 years. ${ }^{12}$ Patients show a lack of keratinisation and pigmentation in the hair accompanied by connective tissue and neurological problems. ${ }^{3}$ The defect in copper homeostasis is caused by mutations in ATP7A, the gene encoding a metal binding ATPase which is thought to be responsible for the appropriate delivery of copper to enzymes and other proteins. ${ }^{4-6}$ The milder disorder, occipital horn syndrome (OHS), in which patients suffer primarily from connective tissue problems, has also been shown to be caused by mutations in ATP7A. However, while the mutations reported in OHS patients are associated with aberrant splicing and the production of some functional $\mathrm{mRNA},{ }^{78}$ mutations reported in classical $\mathrm{MD}$ patients range from large deletions, ${ }^{39}$ single base pair changes, deletions and duplications affecting a few nucleotides, as well as splice site changes, and are often associated with the absence of any functional RNA. $^{810}$

The strong phenotypic, biochemical, and genetic evidence that the mouse mutant mottled $\left(A t p 7 a^{M o}\right)$ is the mouse homologue of Menkes disease has now been supported by the finding of Atp $7 a$ mutations in mottled mice. ${ }^{11-13}$ Affected males are recognised by the presence of curly whiskers, hypopigmentation, and neurological and connective tissue disorders and heterozygous females primarily by the mottled coat. Based on their X linked inheritance pattern and the characteristic phenotype, many mutant alleles, varying considerably in severity, have been recognised as being at Atp $7 a^{\text {Mo }}$. However, allelism is difficult to prove by complementation tests because of the inherent cellular mosaicism associated with $\mathrm{X}$ inactivation. Whereas some alleles, for example, mottled blotchy $\left(A t p 7 a^{\text {Mo-blo }}\right)$, are similar to OHS as affected males are viable and fertile, other alleles, for example, mottled brindled $\left(A t p 7 a^{\text {Mo-br}}\right)$ are associated with liveborn affected males which die before weaning and exhibit similar symptoms to classical $\mathrm{MD}$ patients. ${ }^{14}$

However, over half of the recorded mutants at this locus, for example, mottled dappled and mottled tortoiseshell $\left(A t p 7 a^{M o-d p}\right.$ and $A t p 7 a^{M o-t o}$ ), are associated with prenatal lethality of affected males. This suggests that either mutations in ATP7A may have a more severe effect in mouse than in man, or that there is a strong ascertainment bias for carrier females in the mouse. The mutation spectrum in the two species is different at the molecular level; in a study of 12 different mottled alleles, no deletions or genomic rearrangements were discovered, ${ }^{15}$ whereas in man $20 \%$ of $\mathrm{MD}$ patients have deletions detectable by Southern blotting. ${ }^{9}$ The original report of a partial deletion of Atp7a in mottled dappled was shown to be erroneous, ${ }^{16-18}$ but recently five Atp7a mutations have been reported in mottled alleles. ${ }^{11-13}$ Splice site mutations which remove exons 11 and 14 have been found in mottled blotchy ${ }^{11}$ and mottlec 1 Pub respectively, ${ }^{13}$ amino acid substitutions of residues 1036 and 1364 have been found in mottled viable brindled ${ }^{1213}$ and mottled $11 \mathrm{H}^{13}$ respectively, and a deletion of two amino acids (801 and 802) has been reported in mottled brindled. ${ }^{12}$ These limited data suggest that affected mottled males that die in utero may have mutations which result in a failure to produce any Atp7a protein, whereas those that survive beyond birth may produce protein with a degree of functional activity which is related to the severity of the phenotype. ${ }^{12}{ }^{13}$

For a mouse model to be useful in therapy assessment, it is helpful if the phenotype at all levels is the same as in man. As different mottled alleles give rise to different phenotypes even when maintained on identical genetic backgrounds, it is possible that these phenotypes are related to the immediate effect of the 
mutations on cellular copper kinetics. We therefore wished to examine if any differences in the kinetics of copper homeostasis could be associated with specific mottled alleles and, in particular, if they could be correlated with the phenotypic severity of the allele. In man, copper uptake and retention assays performed on cultured fibroblasts have been used successfully for the pre- and postnatal diagnosis of Menkes disease. ${ }^{19}$ We have applied these assays to primary cell cultures established from nine mouse strains, each of which carried a different mottled mutation and have shown that there is no significant difference in any of the results obtained from the mottled cell cultures or between the mottled cultures and those from $\mathrm{MD}$ patients.

\section{Materials and methods}

MOUSE MUTANTS AND CONTROLS

The nine different alleles of mottled analysed have been described previously. ${ }^{13}$ Four alleles were associated with postnatal survival of affected males; $A t p 7 a^{\text {Mo-blo }}$ males survive and are fertile while mottled $10 \mathrm{H}\left(A t p 7 a^{M o-1 O H}\right)$, mottled 13H $\left(A t p 7 a^{M o-13 H}\right)$, and $A t p 7 a^{M o-b r}$ males die around 10-14 days after birth. The remaining five alleles, $A t p 7 a^{\text {Mo-dp }}$, mottled $11 \mathrm{H}\left(A t p 7 a^{M o-11 H}\right)$, mottled $12 \mathrm{H}$ (Atp7 $a^{\text {Mo-12H}}$ ), mottled $14 \mathrm{H}$ $\left(A t p 7 a^{M o-14 H}\right)$, and $A t p 7 a^{M o-t o}$, are associated with prenatal male lethality and material from heterozygous females was taken for culture and analysis. All mutants were maintained by mating heterozygous females to $3 \mathrm{H} 1$ males, where $3 \mathrm{H} 1$ is a $(\mathrm{C} 3 \mathrm{H} / \mathrm{HeH} \times 101 / \mathrm{H}) \mathrm{F} 1$ hybrid. Control strains of mice represent the different genetic backgrounds on which the various mottled mutants arose ${ }^{14}$ and from 129 (HPRT-), which was used to introduce a selectable $\mathrm{X}$ chromosome into the heterozygotes for carrier studies.

ESTABLISHMENT OF CELL CULTURES

For controls, and alleles associated with postnatal survival of affected males, cultures were initiated either from 19-20 day old embryos or from animals within 24 hours of birth. For alleles associated with prenatal death of affected males, cultures were prepared from heterozygous females as soon as classification was possible, normally one to four days after birth.

Neonates were skinned and eviscerated, the cultures being established from the residual carcass, while embryos were only decapitated. Briefly, each specimen was given $2 \times$ wash with $10 \mathrm{ml}$ PBS in a universal container, transferred to a bijou bottle, and chopped into fragments with fine scissors. After a further $5 \mathrm{ml}$ PBS wash, the fragments were resuspended in $5 \mathrm{ml}$ of normal growth medium (MEM $+15 \%$ FCS, penicillin, and streptomycin) and transferred to a prewetted $75 \mathrm{~cm}^{2}$ flask. Leaving the fragments distributed over the flask surface, the medium was carefully removed; $4 \mathrm{ml}$ of MEM $+50 \%$ FCS was then added, rinsed over the fragments, and removed. Following overnight incubation at $37^{\circ} \mathrm{C}$ to allow the fragments to attach to the flask, $15 \mathrm{ml}$ of normal growth medium was added. After a few days, cells growing out from the fragments were released with trypsin/EDTA and seeded into fresh flasks to create fragment free cultures of fibroblastlike cells.

To try to enrich the heterozygous cultures for cells whose active $\mathrm{X}$ chromosome is that carrying the mutation in Atp $7 a$, matings were set up between heterozygous females and HPRT- males to create $A t p 7 a^{M o} / H p r t$ females. In addition to growth in normal medium, virtually all heterozygous specimens were grown in "HAT" medium (MEM supplemented with $10^{-4} \mathrm{~mol} / 1$ hypoxanthine, $10^{-5} \mathrm{~mol} / 1$ methotrexate, and $1.6 \times 10^{-5} \mathrm{~mol} / 1$ thymidine) for 20-22 days which should have favoured the selection of cells with an active $\mathrm{X}$ chromosome that carried the mutant Atp $7 a$ allele and the normal Hprt allele. However, as there was no significant difference in copper uptake or retention values between heterozygous populations cultured in normal medium or in HAT medium, the selection process was assumed to be ineffective, presumably because of transfer of metabolites between cells. Therefore, the data from these cultures were combined with those from the non-selected cultures.

Aliquots of the different cultures were stored in liquid nitrogen before analysis. Every culture was tested and shown to be free of mycoplasma infection using a PCR method (Stratagene).

COPPER ASSAY

The assay was essentially as in Horn, ${ }^{20}$ but with the addition of the retention measure described in Tønnesen and Horn. ${ }^{19}$ Cultures were grown in test medium for one week, then harvested, seeded in quadruplicate, and incubated for 20 hours in ${ }^{64} \mathrm{Cu}$. Two replicates were sacrificed to measure the uptake of ${ }^{64} \mathrm{Cu}$ into the cells; the remaining two were grown for a further 24 hours in unlabelled medium and the amount of ${ }^{64} \mathrm{Cu}$ retained by the cells was determined.

\section{ANALYSIS OF RESULTS}

Homogeneity of each of the three groups (controls, hemizygotes, and heterozygotes) was tested by a Kruskal-Wallis test, and comparison between the groups was made either by a Mann-Whitney U test (for pairwise comparison) or a Kruskal-Wallis test (for more than two groups).

\section{Results}

Copper uptake (ng ${ }^{64} \mathrm{Cu} / \mathrm{mg}$ protein) and percentage of copper retained were calculated for each of the mottled stocks and controls (table 1). The mean values for these parameters have been calculated for each of the three groups: controls, affected hemizygous males, and heterozygous females. Inspection of the results showed that the uptake of copper is relatively low in the control cultures and, after the 20 hour chase period, they retain less than one quarter of the amount taken up (fig 1). On average, the cultures derived from affected mottled males take up almost six times as much copper as the controls and still retain twothirds of this after the 20 hour chase. The mottled heterozygotes take up more than three 
Table 1 Copper measurements of cell cultures established from mottled and normal mice

\begin{tabular}{|c|c|c|c|c|c|}
\hline \multirow[b]{2}{*}{ Sample } & \multirow[b]{2}{*}{$\mathrm{No}^{\star}$} & \multicolumn{2}{|c|}{$\begin{array}{l}\text { Copper uptake } n g{ }^{64} \mathrm{Cu} / \mathrm{mg} \\
\text { protein }\end{array}$} & \multicolumn{2}{|c|}{$\%$ Copper retained } \\
\hline & & Mean & Range & Mean & Range \\
\hline C57BL/6 & 5 & 14.7 & $12.8-18.1$ & 23.0 & $17.0-28.0$ \\
\hline $3 \mathrm{H} 1$ & 2 & 13.4 & $12.1-14.7$ & 24.0 & $20.7-27.2$ \\
\hline PT & 3 & 13.3 & $12.7-13.8$ & 24.0 & $22.6-26.1$ \\
\hline HPRT & 2 & 17.8 & $15.9-19.7$ & 24.0 & $23.2-24.7$ \\
\hline Controls & 12 & 14.7 & $12.1-19.7$ & 23.5 & $17.0-28.0$ \\
\hline Atp $7 a^{\text {moblo }}$ & 1 & 118.0 & & 86.7 & \\
\hline Atp7 $a^{\text {mobr }}$ & 2 & 79.2 & $63.5-94.9$ & 65.9 & $61.4-70.4$ \\
\hline Atp7 $7 a^{m o l o H}$ & 4 & 75.0 & $51.5-97.4$ & 61.9 & $57.7-74.4$ \\
\hline Atp $7 a^{m 013 H}$ & 2 & 95.1 & $79.7-110.6$ & 70.9 & $61.7-80.1$ \\
\hline Hemizygotes & 9 & 85.2 & $51.5-118.0$ & 67.5 & $57.7-86.7$ \\
\hline Atp $7 a^{\text {modp }}$ & 7 & 58.6 & $29.7-93.0$ & 65.6 & $58.4-78.0$ \\
\hline Atp7 $a^{\text {moto }}$ & 7 & 50.1 & $35.3-58.2$ & 57.8 & $47.4-65.9$ \\
\hline Atp7 $7 a^{m o l l H}$ & 6 & 61.8 & $44.7-77.1$ & 62.2 & $48.2-71.8$ \\
\hline Atp $7 a^{m o l 2 H}$ & 9 & 48.0 & $31.0-53.1$ & 68.5 & $52.9-80.6$ \\
\hline Atp7a $a^{\text {mol4H }}$ & 2 & 43.4 & $33.4-53.3$ & 75.9 & $74.3-77.4$ \\
\hline Heterozygotes & 31 & 52.1 & $29.7-93.0$ & 64.9 & $52.9-80.6$ \\
\hline
\end{tabular}

^Number of cultures tested.

\section{○ Control $\bullet$ Heterozygote $\bullet$ Hemizygote}
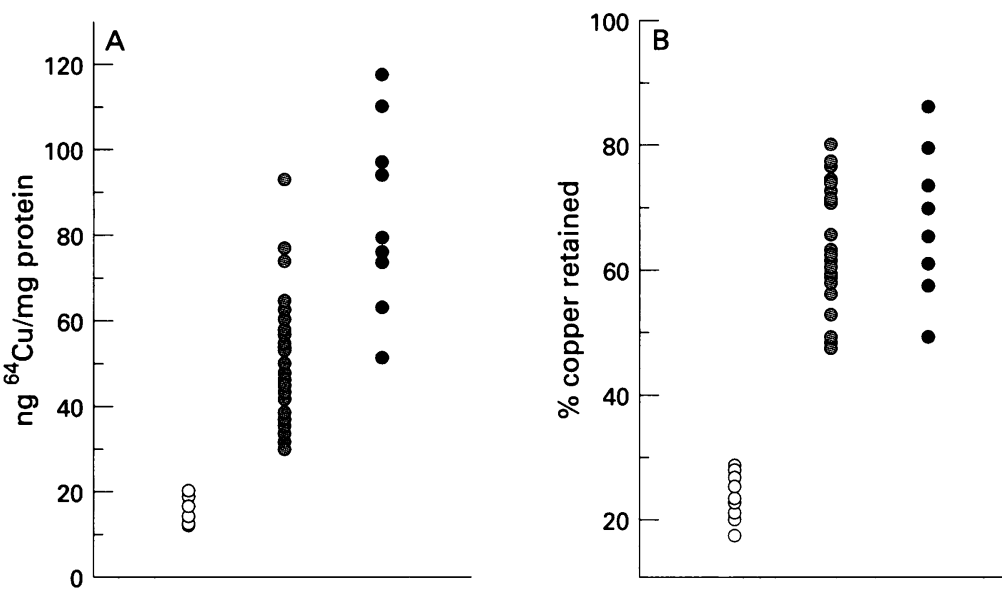

Figure 1 Individual measurements obtained for $(A)$ uptake of ${ }^{64} \mathrm{Cu}$ and $(B)$ percentage of copper retained plotted for each of the three categories, controls, heterozygotes, and hemizygotes. or heterozygote cultures (fig 1). For both uptake and retention of copper the range of values for the control cultures is confined and distinct from the other two classes (in all cases $\mathrm{p}<0.00001)$. In contrast, the other two classes have a large spread of values, with overlap of the upper part of the heterozygote range with the lower part of the hemizygote range for uptake, but almost coincident, slightly less wide, ranges for retention. The hemizygous group is significantly different for copper uptake from the heterozygous group $(u=3.64$, $\mathrm{p}=0.00027$ ) but for retention, the two mutant classes are not significantly different from each other $(u=0.599, p=0.55)$.

\section{Discussion}

Both groups of $A t p 7 a^{\text {Mo }}$ variant cultures, hemizygotes, and heterozygous carriers, were clearly distinguishable from the control cells for uptake and retention of the radiolabelled copper. Within the mutant groups it was not possible to identify any of the mutants as being more or less extreme in the kinetics of copper processing. The only result which showed a marginally significant difference from either of the mutant classes was the retention measure of $A t p 7 a^{M-t o}$, which had a more restricted range and slightly lower mean; nevertheless, the data points fall quite clearly within the heterozygote class. The two $A t p 7 a^{M o}$ groups (hemizygotes and heterozygotes) cannot be compared directly because the heterozygote group is represented by cultures in which there is a mixed phenotype. An absence of metabolic cooperation between the phenotypes was shown by earlier work involving the co-cultivation of varying proportions of human control and Menkes cells which showed a linear relationship between copper uptake and proportion of mutant cells in the population. ${ }^{21}$ That the mouse heterozygotes show a response intermediate between control and hemizygote uptake values would suggest that cellular copper homeostasis, where the mutant allele is active, is similar, irrespective of early lethality. This conclusion is supported by finding that the retention values are the same for both heterozygote and homozygote groups. In the mixed heterozygote population the contribution to copper retention of cells with an active wild type allele is minimal; the average hemizygote cell takes up more than five times as much copper as the wild type and retains three times as much, so to a large extent the retention values are independent of the $\mathrm{X}$ inactivation status of the population. Because the differences in severity seen in the whole organism in the various alleles are not reflected by differences in copper distribution at the cellular level, we suggest that the phenotype must be a reflection of differences in the intracellular distribution of copper.

The range of values reported here for copper uptake in mouse fibroblasts cultured from normal $\left(\sim 15-20 \mathrm{ng}{ }^{64} \mathrm{Cu} / \mathrm{mg}\right.$ protein) and affected males (50-80 ng ${ }^{64} \mathrm{Cu} / \mathrm{mg}$ protein) are almost identical to those reported previously for human fibroblast cultures from OHS and MD patients (normal males and females $\sim 16-30 \mathrm{ng}$
To compare copper uptake and retention measurements between groups, all measurements obtained have been plotted according to whether they came from control, hemizygote, 
${ }^{64} \mathrm{Cu} / \mathrm{mg}$ protein, affected males 50-95 ng ${ }^{64} \mathrm{Cu} / \mathrm{mg}$ protein). ${ }^{1021}$ However, the human and mouse data differ for the heterozygous carriers; the human cultures span the complete range from normal to the maximum value for the affected males, while the mouse results occupy a middle ground. This difference may be explained by the tissue sampling methods used. If tissues are composed of clonal areas, the human samples, which were derived from a relatively small biopsy sample, would have a greater chance of producing cultures biased strongly for the activity of one or other of the alternate $\mathrm{X}$ chromosomes as well as mixed populations. The mouse cells cultured in the present study derive from a major part of the organism, which minimises the possibility of selecting cells with the same $\mathrm{X}$ chromosome active. In a further study, ${ }^{22}$ clones were derived from low numbers of cells sampled from the biopsies of Menkes disease carriers. Most uptake values were either in the normal or the affected range (21 affected, 40 normal, and 13 intermediate). When plotted as a density distribution, cultures from Menkes carriers are clearly skewed towards the control copper uptake values. ${ }^{23}$ The data from cell cultures derived here from $A t p 7 a^{M o}$ heterozygotes do not support a skewing of the phenotype. Because of the limited active lifespan of the mouse cultures it was not possible to conduct cloning tests.

The copper retention part of the assay was devised to provide a prenatal diagnosis for Menkes disease. Copper uptake measured in cultures of cells derived from amniotic fluid was unable to separate the range of "probably affected" male fetuses from that of normals. The first study using a copper retention measure was performed in Menkes fibroblast cell cultures and the introduction of a "chase" period $^{19}$ gave good separation of potentially affected males from controls (affected males $53-84 \%$ retention, normal males $13-23 \%$ retention). These findings were confirmed with amniotic fluid cultures and the retention values obtained are the same as those observed for the mouse cultures studied here.

In conclusion, copper processing measurements have been made on nine cultures established from four independent $A t p 7 a^{M o}$ alleles associated with male survival, 31 cultures established from carriers representing five independent $A t p 7 a^{M o}$ alleles associated with prenatal death of affected males, and 12 control cultures. Both groups of mutants were clearly separable from the controls on both the copper uptake and copper retention assays, and the values obtained were the same as those previously reported for human fibroblasts established from $\mathrm{MD}$ patients and controls. However, despite the varying severity of the phenotype at the level of the whole organism, no significant differences in copper retention were found between alleles at the cellular level.

1 Bankier A. Menkes disease. $\mathcal{F}$ Med Genet 1995;32:213-15.

Horn N, Tønnesen T, Tümer $Z$. Menkes disease: an $\mathrm{X}$-linked neurological disorder of the copper metabolism. Brain Pathol 1992;2:351-62.

3 Danks DM, Campbell P, Stevens BJ, Howell RR. Menkes kinky hair syndrome: an inherited defect of copper absorption with widespread effects. Pediatrics 1962;50:188-201.

4 Chelly J, Tümer Z, Tønnesen $\mathrm{T}$, et al. Isolation of a candidate gene for Menkes disease that encodes a potential heavy metal binding protein. Nat Genet 1993;3:14-19.

5 Mercer JF, Livingston J, Hall B, et al. Isolation of a partial candidate gene for Menkes disease by positional cloning. Nat Genet 1993;3:20-5.

6 Vulpe C, Levinson B, Whitney S, Packman S, Gitschier J. Isolation of a candidate gene for Menkes disease and evidence that it encodes a copper-transporting ATPase. evidence that it encodes

7 Kaler SG, Gallo LK, Proud VK, et al. Occipital horn syndrome and mild Menkes phenotype associated with splice site mutations at the MNK locus. Nat Genet 1994;8 195-202.

8 Das S, Levinson B, Whitney S, Vulpe C, Packman S, Gischier J. Diverse mutations in patients with Menkes disease often lead to exon skipping. Am $\mathcal{F}$ Hum Genet 1994;55 883-9.

9 Tümer Z, Vural B, Tønnesen T, Chelly J, Monaco AP, Horn $\mathrm{N}$. Characterization of the exon structure of the Menkes disease gene using vectorette PCR. Genomics 1995;26:43742.

10 Tümer Z, Lund C, Tolshave J, Burçak V, Tønnesen T, Horn $\mathrm{N}$. Identification of point mutations in 41 unrelated patients affected with Menkes disease. Am $\mathcal{F}$ Hum Genet 1997;60:63-71.

11 Das S, Levinson B, Vulpe C, Whitney S, Gitschier J, Packman S. Similar splicing mutations of the Menkes/mottled copper transporting ATPase gene in occipital horn syndrome and the blotchy mouse. Am f Hum Genet 1995 56:570-6.

12 Reed V, Boyd Y. Mutation analysis provides additional proof that mottled is the mouse homologue of Menkes disease. Hum Mol Genet 1997;6:417-23.

13 Cecchi C, Biasotto M, Tosi M, Avner P. The mottled mouse is a model for human Menkes disease: identification of mutations in the Atp7a gene. Hum Mol Genet 1997;6:42533.

14 Doolittle DP, Davisson MT, Guidi JN, Green MC. Catalogue of mutant genes and polymorphic loci. In: Lyon MF, Rastan S, Brown SDM, eds. Genetic variants and strains of the laboratory mouse. Oxford: Oxford University Press, 1996.

15 George AM, Reed V, Glenister P, et al. Analysis of $M n k$, the murine homologue of the locus for Menkes disease, in normurine homologue of the locus for Menkes diseas,

16 Reed V, Boyd Y. Mutations in mottled dappled are RFLVs. Nat Genet 1994;8:11-12.

17 Levinson B, Vulpe C, Elder B, et al. The mottled gene is the mouse homologue of the Menkes disease gene. Nat Gene 1994;6:369-73.

18 Mercer JFB, Grimes A, Ambrosini L, et al. Mutations in the murine homologue of the Menkes gene in dappled and blotchy mice. Nat Genet 1994;6:374-8.

19 Tønnesen T, Horn N. Prenatal and postnatal diagnosis of Menkes disease, an inherited disorder of copper metabolism. F Inherit Metab Dis 1989;12(suppl 1):207-14.

20 Horn N. Menkes X-linked disease: prenatal diagnosis of hemizygous males and heterozygous females. Prenat Diagn 1989;1:107-20.

21 Horn N. Menkes X linked disease: heterozygous phenotyp in uncloned fibroblast cultures. 7 Med Genet 1980;17:25761.

22 Horn N, Moody P, McGuire VM. Menkes X linked disease: two clonal cell populations in heterozygotes. $7 \mathrm{Med}$ Genet 1980;17:262-6.

23 Horn, N. Menkes X-linked disease: prenatal diagnosis and carrier detection. $\mathcal{F}$ Inherit Metab Dis 1983;6(supp 1):59-62. 